\title{
THE REPRESENTATION OF LINEAR OPERATORS ON SPACES OF FINITELY ADDITIVE SET FUNCTIONS
}

\author{
by C. A. CHENEY and ANDRE de KORVIN \\ (Received 3rd July 1975)
}

\section{Introduction}

The theory of representing continuous linear operators on function spaces in terms of integrals has had a long and fruitful history, beginning with the Riesz representation theorem in 1909. If $T$ is such an operator, then the standard representation is $T(f)=\int f \mathrm{~d} \mu$, where the integral is defined in diverse ways, depending on the nature of the set of functions and the nature of $T$.

However, the theory of representing continuous linear operators on spaces of measures is relatively new. One main interest in such a theory is to try to represent the bidual of $C(X)$, the space of continuous functions on the compact Hausdorff space $X$. It is known that the dual of $C(X)$ is rca $[X, \mathscr{B}]$, the space of all regular countably additive Borel measures on $X$ with finite variation. Thus the bidual of $C(X)$ is the dual of rca $[X, \mathscr{B}]$ and it is desirous to obtain a representation for continuous linear functionals on rca $[X, \mathscr{B}]$.

Mauldin (9) has given a representation for linear operators on rca $[[0,1], \mathscr{B}]$ where $\mathscr{B}$ is the collection of Borel subsets of $[0,1]$. Thus if $T$ is a real-valued continuous operator on rca $[[0,1], \mathscr{B}]$, then $T$ can be written $T(\mu)=\int \psi \mathrm{d} \mu$, where $\psi$ is a real-valued set function defined on $\mathscr{B}$ and the integral is of a special type, sometimes called a KolmogorovBurkill integral. Mauldin states that his methods can be extended to represent linear operators on ca $[S, \Sigma]$, the space of countably additive real-valued set functions defined on a $\sigma$-algebra of subsets of a set $S$, provided the cardinality of $S$ is aleph-one.

Let $C^{* *}(X, E)$ be the bidual of the space of all $E$-valued continuous bounded functions on the normal topological space $X$, where $E$ is a Banach space. Then in (4) it is shown how to represent part of $C^{* *}(X, E)$, identifying the simple function $\chi_{A} \cdot y: X \rightarrow E$ with a particular element in $C^{* *}(X, E)$. Here $y \in E, \chi_{A}$ is the characteristic function of $A$, and $\left(\chi_{A} \cdot y\right)$ $(x)=\chi_{A}(x) \cdot y$.

In other works, operators on spaces of functions of bounded variation have been represented. These are of interest since such spaces are 
sometimes isometrically isomorphic to spaces of finitely additive set functions. See (1), (2), and (6) for details.

In another vein, Gordon (8) has produced an explicit construction of the bidual of the space of real-valued continuous functions on the compact Hausdorff space $X$. The construction uses the fact that $C(X)$ is an $M$-space; the dual of an $M$-space is an $L$-space and the dual of an $L$-space is an $M$-space. Thus the second dual of $C(X)$ can be identified with $C(Y)$ for some compact Hausdorff space $Y$. From $X$, Gordon constructs the required $Y$.

In this paper we consider representing linear operators on spaces of finitely additive set functions, as well as on spaces of countably additive set functions. Our approach is similar to Mauldin's in that the integral used is of Kolmogorov-Burkill type. In the course of the work, we rely on a recent result of Fefferman (7) and on a Lebesgue decomposition theorem for finitely additive set functions due to Darst (3).

Our first result is a representation for linear operators on ba $[S, \Sigma, \lambda]$. It is similar to a recent result of Edwards and Wayment (6) which the authors received while this paper was in progress. However, the KolmogorovBurkill integral used here is perhaps a conceptually simpler integral than the $v$-integral of (6).

We use the first theorem to obtain a representation on all of ba $[S, \Sigma]$. Here an operator is represented as a limit of integrals.

The second section of the paper deals with extensions of the main theorems to representing operators on spaces of vector-valued set functions. Here the range of the set functions is a Banach space $X$ with a particular property which we have called the ARN property, or the approximate Radon-Nikodym property. In a theorem of independent interest, it is shown that every boundedly complete Banach space is an ARN space. Also, every Banach space having the Radon-Nikodym property has the ARN property. Thus the representation theorems hold for a large class of Banach spaces $X$.

\section{Preliminaries}

Throughout, $S$ denotes a non-empty set and $\Sigma$ an algebra of subsets of $S$. Let ba $[S, \Sigma]$ denote all real-valued finitely additive set functions defined on $\Sigma$ having finite variation. Then ba $[S, \Sigma]$ becomes a Banach space under the variation norm. If $\Sigma$ is a $\sigma$-algebra, let ca $[S, \Sigma]$ denote all elements of ba $[S, \Sigma]$ which are countably additive. Then ca $[S, \Sigma]$ is a closed subspace of ba $[S, \Sigma]$.

Let $\lambda: \Sigma \rightarrow X$ and $\mu: \Sigma \rightarrow Y$ be set functions on $\Sigma$ with values in the Banach spaces $X$ and $Y$ respectively. Then $\mu$ is absolutely continuous with respect to $\lambda$, written $\mu \ll \lambda$, if for every $\epsilon>0$, there is a $\delta>0$ such that $\|\mu(A)\|<\epsilon$ whenever $\|\lambda(A)\|<\delta$ and $A \in \Sigma$. In case $\mu$ and $\lambda$ are countably 
additive, this is equivalent to saying $\mu(A)=0$ whenever $\lambda(A)=0$.

If $\lambda \in$ ba $[S, \Sigma]$, let ba $[S, \Sigma, \lambda]$ denote the closed subspace of ba $[S, \Sigma]$ of all elements $\mu$ such that $\mu \ll \lambda$. Similarly, let ca $[S, \Sigma, \lambda]$ denote the closed sub-space of ca $[S, \Sigma]$ of all elements $\mu$ such that $\mu \ll \lambda$.

The integral used in our representation is defined in its general form as follows. Let $X$ and $Y$ be Banach spaces, $L(X, Y)$ the collection of continuous linear operators from $X$ into $Y$, and $\mu: \Sigma \rightarrow X$ and $\Psi: \Sigma \rightarrow$ $L(X, Y)$. Suppose there is an element $y \in Y$ such that for every $\epsilon>0$ there is a partition $\pi_{1}$ of $S$ consisting of measurable sets such that

$$
\left\|\sum_{E_{i} \in \pi}\left\langle\Psi\left(E_{i}\right), \mu\left(E_{i}\right)\right\rangle-y\right\|<\epsilon
$$

for all partitions $\pi$ refining $\pi_{1}$ and consisting of measurable sets. Then $y$ is denoted by $\int \Psi \mathrm{d} \mu$. If $X=$ the real numbers, then $L(X, Y)$ can be identified with $Y$ and $\left\langle\Psi\left(E_{i}\right), \mu\left(E_{i}\right)\right\rangle=\Psi\left(E_{i}\right) \mu\left(E_{i}\right)$.

We now state the results of Fefferman and Darst to be used in this paper.

Let $\pi$ be a partition of $S$ and $\lambda$ be a finitely additive bounded set function on $\Sigma$ into the reals. For each $E \in \Sigma$ define $W_{E}(\cdot)=\lambda_{E}(\cdot) / \lambda(E)$, where $\lambda_{E}(A)=\lambda(A \cap E)$ and quotients of the form $0 / 0$ are taken to be 0 . For $\mu \in$ ba $[S, \Sigma, \lambda]$, Fefferman showed that $\lim _{\pi} \Sigma_{E \in \pi} W_{E}(\cdot) \mu(E)=\mu$ where the limit is in the variation norm as the partitions become finer and finer.

The following Lebesgue decomposition theorem for finitely additive set functions has been proved by Darst (3). For this theorem, the following definition of two such functions being mutually singular is used. Two finitely additive positive set functions $\mu$ and $\lambda$ defined on $\Sigma$ are said to be mutually singular if whenever $A \in \Sigma$ and $\epsilon>0$, then there is a partition $\left\{A_{1}, A_{2}\right\}$ of $A$ such that $\mu\left(A_{1}\right)<\epsilon$ and $\lambda\left(A_{2}\right)<\epsilon$.

Theorem (3). If $\mu$ and $\lambda$ are in ba $[S, \Sigma]$ and $\mu$ and $\lambda$ are both positive, then $\mu=\mu_{a}+\mu_{s}$ where $\mu_{a} \ll \lambda$ and $\mu_{s}$ and $\lambda$ are mutually singular in the above sense. Moreover, this decomposition is unique.

It is not difficult to see that we also have $\mu_{a} \geqslant 0$ and $\mu_{s} \geqslant 0$.

Finally, we give the following definitions. A Banach space $X$ is called boundedly complete if there is a Schauder basis $\left\{x_{n}\right\}$ for $X$ such that $\sup \left\|\sum_{i=1}^{n} a_{i} x_{i}\right\|<\infty$ implies $\sum_{i=1}^{\infty} a_{i} x_{i}$ converges in $X$. For more on boundedly complete spaces, see (10).

A Banach space $X$ is said to have the Radon-Nikodym property if whenever $\mu: \Sigma \rightarrow X, \lambda: \Sigma \rightarrow R, \mu$ and $\lambda$ are countably additive and have finite variation, and $\mu \ll \lambda$, then $\mu(A)=\int_{A} f \mathrm{~d} \lambda$ for some $f \in L_{X}^{\prime}(S, \Sigma, \lambda)$. 


\section{The main results}

We now present our first representation theorem. Let $\lambda$ be a finitely additive, bounded, non-negative set function on $\Sigma$. Let $Y$ be a Banach space.

Theorem 3.1. Let $T:$ ba $[S, \Sigma, \lambda] \rightarrow Y$ be a continuous linear operator. Then there exists a unique finitely additive set function $\Psi: \Sigma \rightarrow Y$ such that $T(\mu)=\int \Psi \mathrm{d} \mu$. Moreover, $\|T\|=\sup _{E \in \Sigma}\|\Psi(E)\|$.

Proof. Using Fefferman's result, we have that $\lim _{\pi} \Sigma_{\pi} W_{E}(\cdot) \mu(E)=\mu$. Thus by the continuity of $T$, we have $\lim _{\pi} T\left(\Sigma_{\pi} W_{E}(\cdot) \mu(E)\right)=$ $\lim _{\pi} \Sigma_{\pi} T\left(W_{E}(\cdot)\right) \mu(E)=T(\mu)$. Thus if we let $T\left(W_{E}(\cdot)\right)=\Psi(E)$, we have $\lim _{\pi} \Sigma_{\pi} \Psi(E) \mu(E)=T(\mu)$. But from the definition of the integral, this means $T(\mu)=\int \Psi \mathrm{d} \mu$.

We now show that $\|T\|=\sup _{E \in \Sigma}\|\Psi(E)\|$. First note that $\operatorname{var}\left[W_{E}\right] \leqslant 1$ since $W_{E}(A)=\lambda(A \cap E) / \lambda(E)$ for all $A \in \Sigma$. So $\left\|T\left(W_{E}(\cdot)\right)\right\|=\|\Psi(E)\| \leqslant$ $\|T\| \operatorname{var}\left[W_{E}\right] \leqslant\|T\|$. Thus $\sup _{E \in \Sigma}\|\Psi(E)\| \leqslant\|T\|$.

On the other hand,

$$
\begin{aligned}
\|T\| & =\sup _{\operatorname{var} \mu \leqslant 1}\|T(\mu)\|=\sup _{\operatorname{var} \mu \leqslant 1}\left\|\int \Psi \mathrm{d} \mu\right\| \\
& =\sup _{\operatorname{var} \mu \leqslant 1}\left\|\lim _{\pi} \sum_{E \in \pi} \Psi(E) \mu(E)\right\|=\sup _{\operatorname{var} \mu \leqslant 1} \lim _{\pi}\left\|\sum_{E \in \pi} \Psi(E) \mu(E)\right\| \\
& \leqslant \sup _{\operatorname{var} \mu \leqslant 1} \lim _{\pi} \sum_{E \in \pi}\|\Psi(E)\| \cdot|\mu(E)| \leqslant \sup _{\operatorname{var} \mu \leqslant 1}\left[\lim _{\pi} \sum_{E \in \pi} \mu(E)\right] \cdot \sup _{E \in \Sigma}\|\Psi(E)\| \\
& \leqslant \sup _{\operatorname{var} \mu \leqslant 1}[\operatorname{var} \mu] \cdot \sup _{E \in \Sigma}\|\Psi(E)\|=\sup _{E \in \Sigma}\|\Psi(E)\| .
\end{aligned}
$$

So $\|T\|=\sup _{E \in \Sigma}\|\Psi(E)\|$. shown.

Using this fact, uniqueness of the representing function $\Psi$ can be

If $T(\mu)=\int \Psi_{1} \mathrm{~d} \mu=\int \Psi_{2} \mathrm{~d} \mu$, then $\int\left(\Psi_{1}-\Psi_{2}\right) \mathrm{d} \mu=0$. Thus $\|0\|=0=$ $\sup _{E \in \Sigma}\left\|\Psi_{1}(E)-\Psi_{2}(E)\right\|$. Thus $\Psi_{1}(E)=\Psi_{2}(E)$ for all $E \in \Sigma$.

Our first theorem represented a linear operator on a set of measures dominated by a fixed measure. We now give a representation for $T$ defined on all of ba $[S, \Sigma]$. To accomplish this, we need the following density theorem which is very similar to a result of Mauldin (9).

Let $H$ be a maximal set of mutually singular non-negative elements of ba $[S, \Sigma]$. Here, mutually singular means in the sense defined above. Let $F$ be the collection of all finite sums of elements in $H$.

Proposition 3.2. The collection $\Gamma$ of all elements in ba $[S, \Sigma]$ which are absolutely continuous with respect to some element in $F$ is dense in ba $[S, \Sigma]$. 
Proof. Suppose first that $\mu \in$ ba $[S, \Sigma]$ and that $\mu \geqslant 0$. Suppose $\lambda_{\alpha} \in F$. Thus $\lambda_{a}=\Sigma \lambda_{i}$ where $\lambda_{i} \in H$ for $i=1, \ldots, n$. Then by the Lebesgue decomposition theorem for finitely additive set functions as proved by Darst, we can write $\mu=\mu_{a}^{\alpha}+\mu_{s}^{\alpha}$ where $\mu_{a}^{\alpha} \ll \lambda_{\alpha}$ and $\mu_{s}^{\alpha}$ and $\lambda_{\alpha}$ are mutually singular. Also, recall from (3) that $\mu_{a}^{\alpha} \geqslant 0$ and $\mu_{s}^{\alpha} \geqslant 0$. Now let $w(\cdot)=\sup \left\{\mu_{a}^{\alpha}(\cdot) \mid \lambda_{\alpha} \in F\right\}$. This collection is directed upward in the sense that if $\lambda_{\alpha}=\Sigma \lambda_{i}$ and $\lambda_{\beta}=\Sigma \lambda_{j}$, then if $\lambda_{\gamma}=\Sigma \lambda_{i}+\Sigma \lambda_{j}$, then $\mu_{a}^{\gamma} \geqslant \mu_{a}^{\alpha}$ and $\mu_{a}^{\gamma} \geqslant \mu_{a}^{\beta}$. Since $\mu_{a}^{\alpha}(\cdot) \leqslant \mu(\cdot)$ for all $\alpha, w(\cdot)$ is finite; furthermore, since ba $[S, \Sigma]$ is a complete lattice and each $\mu_{a}^{\alpha} \in$ ba $[S, \Sigma]$, we must have $w \in$ ba $[S, \Sigma]$.

Now for every $\mu_{\alpha} \in H$, write $\mu=\mu_{a}^{\alpha}+\mu_{s}^{\alpha}$ where $\mu_{a}^{\alpha} \ll \mu_{\alpha}$ and $\mu_{s}^{\alpha}$ and $\mu_{\alpha}$ are mutually singular. Suppose $\mu(E)-w(E)>\mu_{s}^{\alpha}(E)$ for some $E$. Then $\mu(E)=w(E)+(\mu-w)(E)>\mu_{a}^{\alpha}(E)+\mu_{s}^{\alpha}(E)=\mu(E)$, a contradiction. Thus $0 \leqslant \mu-w \leqslant \mu_{s}^{\alpha}$ for all $\alpha$.

Since $\mu_{s}^{\alpha}$ and $\mu_{\alpha}$ are mutually singular, it can be easily seen that $\mu-w$ and $\mu_{\alpha}$ are mutually singular for all $\mu_{\alpha} \in H$. $H$ being maximal, this implies that $\mu-w=0$. Thus $\mu=w$. Thus for every $\epsilon>0$, we can find some $\mu_{\epsilon}$ in $\Gamma$ such that $0 \leqslant \mu(S)-\mu_{\epsilon}(S)=\operatorname{var}\left[\mu-\mu_{\epsilon}\right]<\epsilon$.

Now if $\lambda_{\alpha} \in F$, then $\lambda_{\alpha}=\Sigma \lambda_{i}$ where $i \in I$, a finite set of subscripts. Denoting $\mu_{a}^{\alpha}$ by $\mu_{a}^{I}$, we have shown then that the net $\left\{\mu_{a}^{I}\right\}_{I}$ converges to the element $\mu$ of ba $[S, \Sigma]$ in variation. The preceding was for the case $\mu \geqslant 0$. For any $\mu \in$ ba $[S, \Sigma], \mu$ can be decomposed into positive and negative parts which are still in ba $[S, \Sigma]$. Applying the preceding argument to both parts, we have that $\mu$ is the limit in variation of a net $\left\{\mu_{a}^{I}\right\}_{I}$ where each $\mu_{a}^{I}$ is in $\Gamma$ and thus $\mu_{a}^{I} \ll \Sigma \lambda_{i}$ where the sum ranges over $I$ and each $\lambda_{i}$ is in $H$.

Using the above density theorem, we now prove the following theorem.

Theorem 3.3. Suppose that $T:$ ba $[S, \Sigma] \rightarrow Y$ is a continuous linear operator. Then $T(\mu)=\lim _{1} \int \Psi^{I} \mathrm{~d} \mu_{a}^{I}$, where $\Psi^{I}: \Sigma \rightarrow Y$, and $\mu_{a}^{I}$ are as in the preceding propositions.

Proof. Suppose $\mu \in$ ba $[S, \Sigma]$. By the density theorem we have $\lim _{I} \mu_{a}^{I}=\mu$. By continuity of $T, \lim _{I} T\left(\mu_{a}^{I}\right)=T(\mu)$. Now $\mu_{a}^{I} \ll \Sigma \lambda_{i}$, the sum being taken over $I$. By Theorem 3.1, considering $T$ restricted to ba $\left[S, \Sigma, \Sigma \lambda_{i}\right]$ we can write $T\left(\mu_{a}^{I}\right)=\int \Psi^{I} \mathrm{~d} \mu_{a}^{I}$ where $\Psi^{I}(E)=$ $T\left(\left\{\Sigma\left(\lambda_{i}\right)_{E}(\cdot)\right\} /\left\{\Sigma \lambda_{i}(E)\right\}\right)$. Thus $T(\mu)=\lim _{I} \int \Psi^{I} \mathrm{~d} \mu_{a}^{I}$.

Remarks. 1. We might rephrase this result in terms of projection mappings as follows. For the index $I$, define $P_{I}$ : ba $[S, \Sigma] \rightarrow$ ba $\left[S, \Sigma, \Sigma \lambda_{i}\right]$ by $P_{I}(\mu)=\mu_{a}^{I}$. Then $T(\mu)=\lim _{I} \int \Psi^{I} \mathrm{~d} P_{I}(\mu)$.

2. If ba $[S, \Sigma]$ is replaced by ca $[S, \Sigma]$ in the above theorem, we can obtain a better representation as follows. The decomposition $\mu=\mu_{a}^{I}+\mu_{s}^{I}$ is the usual Lebesgue decomposition. Now for any measure $\mu$ let supp $\mu$ be the set on which $\mu$ is concentrated. Then we have ( $\left.\operatorname{supp} \mu_{s}^{I}\right) \cap$ 
$\left(\cup \operatorname{supp} \lambda_{i}\right)=\emptyset$. Then we have $\int \Psi^{I} \mathrm{~d} \mu=\int \Psi^{I} \mathrm{~d}\left(\mu_{a}^{I}+\mu_{s}^{I}\right)=\int \Psi^{I} \mathrm{~d} \mu_{a}^{I}$ since supp $\Psi^{I} \subset \cup\left(\operatorname{supp} \lambda_{i}\right)$.

Thus $T(\mu)=\lim _{I} \int \Psi^{I} \mathrm{~d} \mu$.

3. If $\nu \in$ ba $\left[S, \Sigma, \Sigma_{I} \lambda_{i}\right]$, then $T(\nu)=j \Psi^{I} \mathrm{~d} \nu$.

4. Our representation depends on the original maximal set $H$ and thus is not unique.

\section{Linear operators on sets of vector-valued set functions}

In this final section we consider some extensions of the above representation theorems. We first establish some notation.

If $X$ is a Banach space, let ba $[S, \Sigma, X]$ denote all finitely additive $X$-valued set functions on $\Sigma$ with finite variation. We point out here that this is not the usual meaning for ba $[S, \Sigma, X]$ : as in (5), this usually denotes the collection of all finitely additive $X$-valued set functions on $\Sigma$ with finite semivariation. If $\lambda$ is a non-negative finitely additive set function on $\Sigma$, we write ba $[S, \Sigma, \lambda, X]$ to denote that subspace of ba $[S, \Sigma, X]$ of elements absolutely continuous with respect to $\lambda$. That is, $\mu$ is in ba $[S, \Sigma, \lambda, X]$ iff for every $\epsilon>0$, there is a $\delta>0$ such that $\|\mu(E)\|<\epsilon$ whenever $\lambda(E)<\delta$.

We will obtain representation theorems for linear operators on ba $[S, \Sigma, \lambda, X]$ and on ca $[S, \Sigma, X]$ when $X$ is a special type of Banach space. We now define the class of Banach spaces which we will use.

Definition. The Banach space $X$ is said to have the approximate Radon-Nikodym property (or $X$ is said to be an ARN space) if whenever $\Sigma$ is a $\sigma$-algebra on a set $S, \lambda: \Sigma \rightarrow R$ is non-negative, finitely additive and with finite variation, and $\mu: \Sigma \rightarrow X$ is finitely additive with $\mu \ll \lambda$, then there is a sequence of $\lambda$-integrable simple functions $f_{n}: S \rightarrow X$ such that $f_{n} \cdot \lambda$ converges to $\mu$ in variation. (Here, $f_{n} \cdot \lambda$ is the $X$-valued set function defined by $f_{n} \cdot \lambda(E)=\int_{E} f_{n} \mathrm{~d} \lambda$.)

In (7) Fefferman showed that $R$ is an ARN space. The following theorem, which is of independent interest, shows that we have many more ARN spaces.

Theorem 4.1. (a) If $X$ is a boundedly complete Banach space, then $X$ is an ARN space.

(b) If $X$ has the Radon-Nikodym property, then $X$ is an ARN space.

Proof. (a) Suppose $X$ is boundedly complete and that $S$ and $\Sigma$ are given. Also suppose that $\mu$ and $\lambda$ are given as in the definition of ARN space.

We begin with an idea used in Uhl [11]. Let $\tau: \Sigma \rightarrow \hat{\Sigma}$ be the Boolean isomorphism where $\hat{\Sigma}$ is the algebra of all compact open subsets of a totally disconnected compact Hausdorff space $\hat{S}$. Let $\hat{\lambda}(\tau(E))=\lambda(E)$ and $\hat{\mu}(\tau(E))=\mu(E)$. Then $\hat{\lambda}$ is regular. By Alexandroff's theorem $(5$, p. 138) $\hat{\lambda}$ is countably additive. Let $\hat{\Sigma}_{0}$ be the $\sigma$-algebra generated by $\hat{\Sigma}$. Then 
consider $\hat{\Sigma}_{0}(\hat{\Lambda})$, the collection of (equivalence classes of) sets in $\hat{\Sigma}_{0}$ with the metric $d\left(E_{1}, E_{2}\right)=\hat{\lambda}\left(E_{1} \Delta E_{2}\right), E_{i} \in \hat{\Sigma}_{0}$. Then $\hat{\Sigma}$ is a dense subspace of the metric space $\hat{\Sigma}_{0}(\hat{\lambda})$ and we have the following identities:

$$
\begin{gathered}
\hat{\lambda}\left(E_{1} \Delta E_{2}\right)=\hat{\lambda}\left(E_{1}-E_{2}\right)+\hat{\lambda}\left(E_{2}-E_{1}\right) \\
\hat{\mu}\left(E_{1}\right)-\hat{\mu}\left(E_{2}\right)=\hat{\mu}\left(E_{1}-E_{2}\right)-\hat{\mu}\left(E_{2}-E_{1}\right)
\end{gathered}
$$

for all $E_{i}$ in $\hat{\Sigma}$.

These facts together with the knowledge that $\hat{\mu} \ll \hat{\lambda}$ on $\hat{\Sigma}$ imply that $\hat{\mu}$, considered as a function on $\hat{\Sigma} \subset \hat{\Sigma}_{0}(\hat{\lambda})$, is uniformly continuous and that $\hat{\mu}$ has a $\hat{\lambda}$-continuous extension to all of $\hat{\Sigma}_{0}(\hat{\lambda})$. So we have $\hat{\lambda}: \hat{\Sigma}_{0}(\hat{\lambda}) \rightarrow R$ countably additive and regular and $\hat{\mu}: \hat{\Sigma}_{0}(\hat{\lambda}) \rightarrow X$.

Now if $\left\{x_{n}\right\}$ is the Schauder basis for $X$, we can write $\hat{\mu}(E)=$ $\sum_{n=1}^{\infty} \hat{\mu}_{n}(E) x_{n}$ for every $E$ in $\hat{\Sigma}_{0}(\hat{\lambda})$. Then each $\hat{\mu}_{n}$ is a real-valued countably additive regular set function on $\hat{\Sigma}_{0}(\hat{\lambda})$. Also, $\hat{\mu}_{n} \ll \hat{\lambda}$ for every $n$. Thus we have $\hat{\mu}_{n}(\cdot)=\int_{(-)} \hat{f}_{n} \mathrm{~d} \hat{\lambda}$ for some $\hat{\lambda}$-integrable function $\hat{f}_{n}$.

We shall now show that if $\hat{P}_{n}(\cdot)=\sum_{i=1}^{n} \hat{f}_{i}(\cdot) x_{i}$, then $\lim _{n} \hat{P}_{n}=\hat{P}$ exists in the norm of $L^{\prime}(\hat{S}, \hat{\lambda}, X)$ and $\hat{\mu}(\cdot)=\lim _{n} \int_{(\cdot)} \hat{P}_{n} \mathrm{~d} \hat{\lambda}=\int_{(\cdot)} \hat{P} \mathrm{~d} \hat{\lambda}$ where the limit is in the variation norm.

Since $\left\{x_{n}\right\}$ is a Schauder basis for $X$, there exists a constant $K>0$ such that

$$
\left\|\sum_{i=1}^{n} a_{i} x_{i}\right\| \leqslant K\left\|\sum_{i=1}^{\infty} a_{i} x_{i}\right\| \text { for all } n
$$

whenever the right hand side exists. Now since var $\hat{\mu} \ll \hat{\lambda}$ and var $\hat{\mu}<\infty$, we have $\operatorname{var} \hat{\mu}(\cdot)=\int_{(\cdot)} g \mathrm{~d} \hat{\lambda} \leqslant \int_{S} g \mathrm{~d} \hat{\lambda}<\infty$ where $g(t) \geqslant 0 \hat{\lambda}$-almost everywhere on $\hat{S}$ and $g$ is $\hat{\lambda}$-integrable over $\hat{S}$. Let $\hat{Q}_{n}(E)=\sum_{i=1}^{n} \hat{\mu}_{i}(E) x_{i}$ for all $E$ in $\hat{\Sigma}_{0}(\hat{\lambda})$. Then we have var $\hat{Q}_{n}(E) \leqslant K \cdot \operatorname{var} \hat{\mu}(E)$ from $(1)$. Thus since $\hat{P}_{n}(t)$ is the Radon-Nikodym derivative of $\hat{Q}_{n}$, we have $\left\|\hat{P}_{n}(t)\right\| \leqslant K g(t)$ for all $n$ and $\hat{\lambda}$-almost everywhere. Since $X$ is boundedly complete, this implies that $\lim _{n} \hat{P}_{n}(t)=\hat{P}(t)$ exists for almost all $t$ in $\hat{S}$. From the last inequality and the Lebesgue dominated convergence theorem we conclude that $\hat{P}_{n}$ converges to $\hat{P}$ in $L^{1}(\hat{S}, \hat{\lambda}, X)$ norm. Thus we have that

$$
\hat{\mu}(\cdot)=\lim _{n} \hat{Q}_{n}(\cdot)=\lim _{n} \int_{(\cdot)} \hat{P}_{n} \mathrm{~d} \hat{\lambda}=\int_{(\cdot)} \hat{P} \mathrm{~d} \hat{\lambda}
$$

where the limit is in the variation norm.

Thus it is possible to find simple functions $\hat{s}_{n}: \hat{S} \rightarrow X$ such that $\int_{(\cdot)} \hat{s}_{n} \mathrm{~d} \hat{\lambda}$

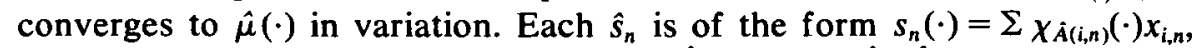
the sum ranging over $i=1, \ldots, k_{n}$, where $\hat{A}(i, n)$ is in $\hat{\Sigma}_{0}(\hat{\lambda})$, for every fixed $n$ the $\hat{A}(i, n)$ are mutually disjoint, and $x_{i, n} \in X$.

By the regularity of $\hat{\lambda}$, each $\hat{A}(i, n)$ could be taken to be in $\hat{\Sigma}$.

So there exist $\hat{A}(i, n)$ in $\hat{\Sigma}$ and $x_{i, n}$ in $X$ such that for every $\epsilon>0$,

$$
\sum\left\|\hat{\mu}\left(\hat{E}_{j}\right)-\sum \lambda\left(\hat{A}(i, n) \cap \hat{E}_{j}\right) x_{i, n}\right\|<\epsilon
$$


for $n$ large enough, where the inner sum ranges over $i=1, \ldots, k_{n}$ and the outer sum is taken over a partition $\left\{\hat{E}_{j}\right\}$ of $\hat{S}$. Letting $A=\tau^{-1}(\hat{A})$ for every $\hat{A} \in \hat{\Sigma}$, since $\tau$ is an isomorphism we can rewrite the above inequality as

$$
\sum\left\|\mu\left(E_{j}\right)-\sum \lambda\left(A(i, n) \cap E_{j}\right) x_{i, n}\right\|<\epsilon
$$

for $n$ large enough, where the inner sum ranges over $i=1, \ldots, k_{n}$ and the outer sum is now taken over a partition $\left\{E_{j}\right\}$ of $S$. Thus if we let $s_{n}(\cdot)=\Sigma \chi_{\mathrm{A}(i, n)}(\cdot) x_{i, n}$, the sum over $i=1, \ldots, k_{n}$, we have shown that $\int s_{n} \mathrm{~d} \lambda$ converges to $\mu(\cdot)$ in variation. We have thus shown that $X$ is an ARN space and this completes the proof of part (a).

(b) We use the notation of the proof in part (a). Since $X$ has the Radon-Nikodym property, there exists a $\hat{\lambda}$-integrable function $\hat{f}$ such that $\hat{\mu}(\cdot)=\int_{(\cdot)} \hat{f} \mathrm{~d} \hat{\lambda}$. Then we can obtain a sequence of $\hat{\lambda}$-integrable simple functions $\hat{s}_{n}$ such that $\lim \hat{s}_{n}=\hat{f}$ in the $L^{1}$ norm. Then $\hat{f}_{(,)} \hat{s}_{n} \mathrm{~d} \hat{\lambda}$ converges to $\hat{\mu}(\cdot)$ in variation norm, and the proof then proceeds as in the proof of part (a).

We now give a proposition needed in our next representation theorem. Suppose $\mu \ll \lambda$, where $\mu$ and $\lambda$ are as in the definition of ARN space.

Proposition 4.2. Suppose $X$ is an ARN space. Let $s_{\pi}=$ $\Sigma\{\mu(E) / \lambda(E)\} \chi_{E}$ where the sum is taken over a partition $\pi$ of $S$ into measurable sets $E$. Then $\lim _{\pi} s_{\pi} \cdot \lambda=\mu$ in variation.

Proof. We first recall that $s_{\pi} \cdot \lambda$ is the set function defined by $s_{\pi} \cdot \lambda(\cdot)=\int_{(\cdot)} s_{\pi} \mathrm{d} \lambda$. Now let $\epsilon>0$. Choose a simple function $s_{1}: S \rightarrow X$ such that $\operatorname{var}\left[\mu-\mu_{1}\right]<\epsilon / 2$, where $\mu_{1}(\cdot)=\int_{(\cdot)} s_{1} \mathrm{~d} \lambda$. We can find such a function since $X$ is an ARN space.

Now if $S_{1}=\Sigma_{\chi_{E(i)} y_{i}}$ where the sum is taken over $i=1, \ldots, n$, the $E(i)$ are disjoint and $y_{i} \in X$, then we have $y_{i}=\mu_{1}(E(i) / \lambda(E(i))$.

If $\left\{E(j)^{\prime}\right\}$ is a refinement of $\{E(i)\}$, then we have

$$
s_{1}=\Sigma\left\{\mu_{1}\left(E(j)^{\prime}\right) / \lambda\left(E(j)^{\prime}\right)\right\} \chi_{E\left(j^{\prime}\right.}
$$

where the sum ranges over $j=1, \ldots, m$. Now let $\pi$ be any refinement of $\{E(i)\}$. Then

$$
\begin{aligned}
\operatorname{var}\left[\mu_{1}-s_{\pi} \cdot \lambda\right] & =\operatorname{var}\left[s_{1} \cdot \lambda-s_{\pi} \cdot \lambda\right] \\
& =\sum \int_{E}\left\|\left\{\mu_{1}(E) / \lambda(E)\right\}-\{\mu(E) / \lambda(E)\}\right\| \mathrm{d} \lambda \\
& =\sum\left\|\mu_{1}(E)-\mu(E)\right\| \leqslant \operatorname{var}\left[\mu_{1}-\mu\right]<\epsilon / 2
\end{aligned}
$$

where the sums are taken over $E$ in $\pi$. Thus we have $\operatorname{var}\left[\mu-s_{\pi} \cdot \lambda\right]<\epsilon$. So $\lim _{\pi} s_{\pi} \cdot \lambda=\mu$ in variation. 
We now give the following representation theorem.

Theorem 4.3. Suppose $T$ : ba $[S, \Sigma, \lambda, X] \rightarrow Y$ is a continuous linear operator where $Y$ is a Banach space and $X$ is an ARN space. Then there exists a unique $\mu$-integrable function $\Psi: \Sigma \rightarrow L(X, Y)$ such that $T(\mu)=$ $\int \Psi \mathrm{d} \mu$. Furthermore, $\|T\|=\sup \|\Psi(E)\|$, the sup being taken over all $E \in \Sigma$.

Proof. For any partition $\pi$ of $S$ into measurable sets, let $P_{\pi}(\mu)(\cdot)=$ $\int_{(\cdot)} \Sigma\{\mu(E) / \lambda(E)\}_{X_{E}} \mathrm{~d} \lambda$, where the sum is over $E$ in $\pi$. Then by the above proposition, $P_{\pi}(\cdot)$ converges to $\mu$ in variation. For every $x \in X$ and $E \in \Sigma$ the set function $W_{E, x}(\cdot)=\left\{\lambda_{E}(\cdot) / \lambda(E)\right\} x$ is an element of ba $[S, \Sigma, \lambda, X]$. Thus

$$
\begin{aligned}
T\left(P_{\pi}(\mu)\right) & =\sum T\left[f_{(\cdot)}\{\mu(E) / \lambda(E)\}_{\chi_{E}} \mathrm{~d} \lambda\right]=\sum T\left[\{\mu(E) / \lambda(E)\} \lambda_{E}(\cdot)\right] \\
& =\sum T\left[\left\{\lambda_{E}(\cdot) / \lambda(E)\right\} \mu(E)\right]=\sum\langle\Psi(E), \mu(E)\rangle
\end{aligned}
$$

where $\langle\Psi(E), x\rangle=T\left[\left\{\lambda_{E}(\cdot) / \lambda(E)\right\} x\right]$ for every $x \in X$ and where the sums are taken over all $E$ in $\pi$. Thus $\Psi(E)$ is a linear operator on $X$ with values in $Y$. It is not hard to verify that $\Psi(E)$ is continuous. Thus $\Psi: \Sigma \rightarrow L(X, Y)$. Thus we have shown that $\lim _{\pi} T\left(P_{\pi}(\mu)\right)=\lim _{\pi} \Sigma\langle\Psi(E), \mu(E)\rangle=T(\mu)$, the sum being taken over $E$ in $\pi$. By our definition of the integral this means that $T(\mu)=\int \Psi \mathrm{d} \mu$. The fact that $\|T\|=\sup \|\Psi(E)\|$, the sup being taken over $E$ in $\Sigma$, is proved analogously as in Theorem 3.1.

For our final theorem, we give a representation for operators on ca $[S, \Sigma, X]$ which are continuous in a certain topology on ca $[S, \Sigma, X]$. We define this topology by net convergence:

$$
\lim _{\alpha} \mu_{\alpha}=\mu \text { iff } \lim _{\alpha} \operatorname{var}\left[x^{*} \mu_{\alpha}-x^{*} \mu\right]=0 \text { for every } x^{*} \in X^{*} .
$$

Here, of course, $x^{*} \mu_{\alpha}$ and $x^{*} \mu$ are in ca $[S, \Sigma]$. Suppose now that ca $[S, \Sigma, X]$ is so topologised and that $X$ is an ARN space.

Theorem 4.4. Suppose $T: \mathrm{ca}[S, \Sigma, X] \rightarrow Y$ is a continuous linear operator. Then $T(\mu)=\lim _{I} \int \Psi^{I} \mathrm{~d} \mu^{I}$, where $I$ is a finite set of indices of elements in $H, \mu^{I} \ll \Sigma \lambda_{\alpha}$ (the sum being taken over $I$ ), and $\Psi^{I}: \Sigma \rightarrow$ $L(X, Y)$.

Proof. Recall the definition of $H$ given immediately before Proposition 3.2. Let $\lambda=\Sigma \lambda_{\alpha}$, the sum taken over $I$, and $\mu=\mu^{I}+\mu_{I}$ be the Lebesgue decomposition of $\mu$ with $\mu^{I} \ll \lambda$ and $\mu_{I}$ mutually disjoint from $\lambda$. For $x^{*}$ in $X^{*}, x^{*} \mu=x^{*} \mu_{I}+x^{*} \mu^{3}$. Now the scalar measure $x^{*} \mu$ has a Lebesgue decomposition with respect to $\lambda: x^{*} \mu=\left(x^{*} \mu\right)_{t}+\left(x^{*} \mu\right)^{I}$ where $\left(x^{*} \mu\right)^{I} \ll \lambda$ and $\left(x^{*} \mu\right)_{I}$ and $\lambda$ are mutually disjoint. By the uniqueness of Lebesgue decomposition we have $x^{*} \mu^{I}=\left(x^{*} \mu\right)^{I}$. By Proposition 3.2 we have that $\lim _{I} x^{*} \mu^{I}=x^{*} \mu$. Thus $\lim _{I} \mu^{I}=\mu$ in the topology for 20/3-E 
ca $[S, \Sigma, X]$. Thus $\lim _{I} T\left(\mu^{I}\right)=T(\mu)$. But from Theorem 4.3, considering $T$ restricted to ba $[S, \Sigma, \lambda, X]$, we have $T\left(\mu^{I}\right)=\int \Psi^{I} \mathrm{~d} \mu^{I}$ where $\Psi^{I}: \Sigma \rightarrow$ $L(X, Y)$. So $T(\mu)=\lim _{I} \int \Psi^{I} \mathrm{~d} \mu^{I}$.

\section{Acknowledgment}

We wish to thank the referee for his helpful suggestions, especially those leading to the present form of the proof of Theorem 4.1.

\section{REFERENCES}

(1) R. A. Alo and A. DE KORVIN, Representation theorems for linear operators, Proceedings of the Conference on Functional Analysis and its Applications, Kanpur, India, pp. 1-12.

(2) R. A. Alo, A. DE KORVIN and R. EASTON, Functions of bounded variation on idempotent semigroups, Math. Ann. 194 (1971), 1-11.

(3) R. DARST, A decomposition for finitely additive set functions, J. Reine Angew. Math. 210 (1962), 31-37.

(4) A. DE KORVIN and R. EASTON, Some representation theorems, Rocky Mountain J. Math. 1 (1971), 561-572.

(5) N. DUNFORD and J. SCHWARTZ, Linear Operators, Part I (New York, Interscience, 1958).

(6) J. EDWARDS and S. WAYMENT, Extensions of the v-integral, Trans. Amer. Math. Soc. 191 (1974), 165-184.

(7) C. FEFFERMAN, A Radon-Nikodym theorem for finitely additive set functions, Pacific J. Math. 23 (1967), 35-45.

(8) H. GoRDON, The maximal ideal space of a ring of measurable functions, Amer. J. Math. 88 (1966), 827-843.

(9) D. MAULdiN, A representation for the second dual of $C[0,1]$, Studia Math. (to appear).

(10) I. SINGER, Bases in Banach Spaces (Springer-Verlag, New York, 1970).

(11) J. UHL, Extensions and decompositions of vector measures, J. London Math. Soc. 3 (1971), 672-676.

DePaRTMENT OF MATHEMatics

INDIANA STATE UNIVERSITY

TERRE HAUTE, INDIANA 47809 